\title{
On Local Lyapunov Exponents of Chaotic Hamiltonian Systems
}

\author{
Timo Hofmann, Jochen Merker* \\ Faculty of Computer Science, Mathematics and Natural Sciences \\ PF 3011 66, 04251 Leipzig, Germany \\ *E-mail: jochen.merker@htwk-leipzig.de
}

Received: 31 December 2017; revised: 27 March 2018; accepted: 05 April 2018; published online: 30 May 2018

\begin{abstract}
Chaos in conservative systems, particularly in Hamiltonian systems, is different from chaos in dissipative systems. For example, not only the eigenvalues of the symmetric Jacobian, but also the global Lyapunov exponents of Hamiltonian systems occur in pairs $(\lambda,-\lambda)$. In this article, we even show that appropriately defined local Lyapunov exponents occur in pairs, and in turn this allows to give a new and easily accessible proof of the pairing property for global Lyapunov exponents. As examples of low dimensional chaotic Hamiltonian systems, we discuss the classical Hénon-Heiles system and a sixth order generalisation. For the latter, there is numerical evidence of two disjoint chaotic seas.
\end{abstract}

Key words: Lyapunov exponents, Hamiltonian systems, chaos, symplectic, Iwasawa decomposition

\section{INTRODUCTION}

This article aims to discuss certain aspects of chaos in Hamiltonian systems and tries to answer some of the questions posed in [9].

One property of Hamiltonian systems is that the eigenvalues of the symmetric Jacobian as well as the global Lyapunov exponents occur in pairs $(\lambda,-\lambda)$. This property has been known for a long time and has been proved in [2], see also [1]. For covariant local Lyapunov exponents of Hamiltonian systems, the same property has been claimed in [11], but a general proof of this claim does not seem to be known in literature. To clarify this situation, [9] asks to devise relatively simple autonomous Hamiltonian systems for which long-time local pairing is absent. We prove that there are no such systems, because appropriately defined local Lyapunov exponents always occur in pairs $(\lambda,-\lambda)$.

More precisely, all continuous functions $\lambda_{i}(\cdot)$ on state space with the property that the time average $\frac{1}{t} \int_{0}^{t} \lambda_{i}(x(s)) d s$ converges to the $i$-th Lyapunov exponent of the orbit $x(t)$ for $i=1, \ldots, n$ deserve to be called local Lyapunov exponents in a wide sense. With this general definition, local Lyapunov exponents obviously do not need to satisfy the pairing pro- perty. Yet, there are certain methods to define local Lyapunov exponents, and the question occurs whether the so defined local Lyapunov exponents have the pairing property. We show that the local Lyapunov exponents defined via the QR method generally do not have the pairing property, while the symplectic local Lyapunov exponents defined by a symplectic modification of the QR method using the Iwasawa decomposition and the SVD local Lyapunov exponents defined via singular value decomposition have the pairing property. The latter are related to covariant local Lyapunov exponents.

\section{Outline}

In Section II.1 we recall basic notions related to autonomous ODEs and their linearization. Afterwards, we discuss eigenvalues of the symmetric Jacobian, global Lyapunov exponents and local Lyapunov exponents - particularly the QR method - in the general situation, where the system is not necessarily Hamiltonian. In Section II.5 we introduce Hamiltonian systems on linear Poisson manifolds.

Section III is the main theoretical part of this article, where the pairing property is proved for symplectic local Lyapunov exponents and SVD local Lyapunov exponents, 
which are related to covariant local Lyapunov exponents.

Section IV contains a numerical study of two fourdimensional chaotic Hamiltonian systems, the classical Hénon-Heiles system and a sixth order generalisation. For the classical Hénon-Heiles system a numerical example is given that the QR local Lyapunov exponents do not have the pairing property, while the symplectic local Lyapunov exponents and the SVD local Lyapunov exponents always have the pairing property. For the sixth order generalization numerical evidence is presented that there are two distinct chaotic seas at energy $H=18$.

\section{PRELIMINARIES}

\section{1. Autonomous ODEs}

An equation of the form

$$
\dot{x}(t)=f(x(t))
$$

with a continuous vector field $f$ on the real vector space $\mathbb{R}^{n}$ is called an autonomous ordinary differential equation (ODE). If there exists locally in time a unique solution of (1) to every initial value, then a local flow $\Phi$ on $\mathbb{R}^{n}$ is generated by $\Phi\left(t, x_{0}\right):=x(t)$, where $x(t)$ denotes the solution to the initial value $x(0)=x_{0}$.

To estimate how perturbations affect a solution $x(t)$ of (1), let us assume that $f$ is continuously differentiable and consider the linearization

$$
\dot{y}(t)=D f(x(t)) y(t)
$$

of (1) along $x(t)$, where $D f\left(x_{0}\right)$ denotes the Jacobian matrix of $f$ at the state $x_{0}$. If $x(t)$ is the solution of (1) to the initial value $x(0)=x_{0}$, then due to linearity the solution of (2) to an arbitrary initial perturbation $y_{0}$ can be written as $y(t)=Y\left(t, x_{0}\right) y_{0}$ with a matrix-valued function $Y$ depending on time $t$ and the initial state $x_{0}$. Hereby, $Y\left(t, x_{0}\right)$ is the solution of the matrix $\operatorname{ODE} \frac{d}{d t} Y\left(t, x_{0}\right)=D f(x(t)) Y\left(t, x_{0}\right)$ associated with (2) to the initial value $Y\left(0, x_{0}\right)=\operatorname{Id}$, and $Y$ satisfies the cocycle condition

$$
Y\left(t+s, x_{0}\right)=Y(s, x(t)) Y\left(t, x_{0}\right),
$$

where $x(t)$ denotes the solution of (1) to the initial value $x(0)=x_{0}$ at time $t$.

\section{2. Eigenvalues of the symmetric Jacobian}

While the Jacobian matrix $D f\left(x_{0}\right)$ of $f$ at $x_{0}$ may have complex eigenvalues, every eigenvalue of the symmetric Jacobian matrix $D f\left(x_{0}\right)_{\mathrm{sym}}:=\frac{1}{2}\left(D f\left(x_{0}\right)+D f\left(x_{0}\right)^{T}\right)$ is real. When counted with algebraic multiplicity, there are $n$ real eigenvalues $\mu_{i}\left(x_{0}\right), i=1, \ldots, n$, of $D f\left(x_{0}\right)_{\text {sym }}$ at $x_{0}$, and these eigenvalues can be ordered so that $\mu_{1}\left(x_{0}\right) \geq$ $\cdots \geq \mu_{n}\left(x_{0}\right)$. The skew-symmetric part $D f\left(x_{0}\right)_{\text {skew }}:=$
$\frac{1}{2}\left(D f\left(x_{0}\right)-D f\left(x_{0}\right)^{T}\right)$ of $D f\left(x_{0}\right)$ has only purely imaginary eigenvalues and does not lead to any growth or decay of the perturbation, as the Euclidean norm $\|y(t)\|_{2}$ of a solution $y(t)$ of (2) depends only on the symmetric part of $D f(x(t))$ due to

$$
\begin{aligned}
\frac{d}{d t}\left(\frac{1}{2}\|y(t)\|_{2}^{2}\right) & =y(t)^{T} D f(x(t)) y(t)= \\
& =y(t)^{T} D f(x(t))_{\mathrm{sym}} y(t)
\end{aligned}
$$

where $y(t)^{T} D f(x(t))_{\text {skew }} y(t)=0$ was used. Thus, the eigenvalues of the symmetric Jacobian matrix $D f\left(x_{0}\right)_{\text {sym }}$ can be interpreted as infinitesimal growth or decay rates of perturbations at the state $x_{0}$. Particularly, if the largest eigenvalue $\mu_{1}(x(t))$ of $D f(x(t))_{\text {sym }}$ along a solution $x(t)$ satisfies $\int_{0}^{\infty} \mu_{1}(x(s)) d s=-\infty$, then (3) implies

$\frac{d}{d t}\left(\frac{1}{2}\|y(t)\|_{2}^{2}\right) \leq 2 \mu_{1}(x(t))\left(\frac{1}{2}\|y(t)\|_{2}^{2}\right) \stackrel{\text { Gronwall's inequality }}{\Longrightarrow}$ $\|y(t)\|_{2} \leq\left\|y_{0}\right\|_{2} \exp \left(\int_{0}^{t} \mu_{1}(x(s)) d s\right) \rightarrow 0$ as $t \rightarrow \infty$.

i.e. perturbations decay to zero as $t \rightarrow \infty$. Conversely, $\mu_{1}>0$ on sufficiently large parts of an orbit is necessary for sensitive dependence on initial conditions and thus for chaotic behaviour.

Note that the eigenvalues of the symmetric Jacobian matrix $D f\left(x_{0}\right)_{\text {sym }}$ are related to the cocycle $Y\left(t, x_{0}\right)$ by the identity

$$
\mu_{i}\left(x_{0}\right)=\lim _{t \rightarrow 0} \frac{1}{t} \ln \left(\left\|Y\left(t, x_{0}\right) y_{i}\right\|_{2}\right)
$$

where $y_{i}$ is a normed eigenvector of $D f\left(x_{0}\right)_{\text {sym }}$ from the eigenspace $E_{\mu_{i}\left(x_{0}\right)}\left(x_{0}\right)$ to the eigenvalue $\mu_{i}\left(x_{0}\right)$. In fact, the limit in (4) is the time derivative of $\ln \left(\left\|Y\left(t, x_{0}\right) y_{i}\right\|_{2}\right)$ at $t=0$, and because

$$
\begin{aligned}
\ln \left(\left\|Y\left(t, x_{0}\right) y_{i}\right\|_{2}\right) & =\frac{1}{2} \ln \left(\left\|Y\left(t, x_{0}\right) y_{i}\right\|_{2}^{2}\right)= \\
& =\frac{1}{2} \ln \left(\left\langle Y\left(t, x_{0}\right)^{T} Y\left(t, x_{0}\right) y_{i}, y_{i}\right\rangle\right)
\end{aligned}
$$

and

$$
\begin{aligned}
& \left.\quad \frac{d}{d t}\left(\frac{1}{2} Y\left(t, x_{0}\right)^{T} Y\left(t, x_{0}\right)\right)\right|_{t=0}=\frac{1}{2}\left(D f\left(x_{0}\right)\right. \\
& \left.+\operatorname{Df}\left(x_{0}\right)^{T}\right)=\operatorname{Df}\left(x_{0}\right)_{\mathrm{sym}}
\end{aligned}
$$

hold, we have

$$
\begin{aligned}
& \lim _{t \rightarrow 0} \frac{1}{t} \ln \left(\left\|Y\left(t, x_{0}\right) y_{i}\right\|_{2}\right)= \\
= & \left\langle D f\left(x_{0}\right)_{\mathrm{sym}} y_{i}, y_{i}\right\rangle=\mu_{i}\left(x_{0}\right)\left\|y_{i}\right\|_{2}^{2}=\mu_{i}\left(x_{0}\right) .
\end{aligned}
$$




\section{3. Global Lyapunov exponents}

The eigenvalues $\mu_{i}\left(x_{0}\right)$ merely capture the infinitesimal growth or decay rates of perturbations at a state $x_{0}$, but not that $Y\left(t, x_{0}\right)$ may rotate an initial perturbation $y_{i}$ from the eigenspace of the eigenvalue $\mu_{i}\left(x_{0}\right)$ into the eigenspace of a different eigenvalue at state $x(t)$. Therefore, the values $\frac{1}{t} \ln \left(\left\|Y\left(t, x_{0}\right) y_{i}\right\|\right)$ at a time $t>0$ may not be identical with $\mu_{i}(x(t))$, even if $y_{i}$ is a normed eigenvector to $\mu_{i}\left(x_{0}\right)$. Further, the distribution given by the eigenspaces $E_{\mu_{i}(x(t))}(x(t))$ along the orbit $x(t)$ is not invariant under $Y\left(t, x_{0}\right)$.

The long time growth or decay rates along the orbit starting at $x_{0}$ are precisely captured by global Lyapunov exponents. These are used in many applications and for many purposes. Most importantly, a positive global Lyapunov exponent implies sensitive dependence on initial conditions and therefore indicates a chaotic behaviour of the system. However, there are many other applications like the Kaplan-Yorke formula, which allows to determine the dimension of the attractor from the global Lyapunov exponents, see [7].

The global Lyapunov exponents $\lambda_{i}$ (also called characteristic exponents) are defined by

$$
\lambda_{i}:=\lim _{t \rightarrow \infty} \frac{1}{t} \log \left(\left\|Y\left(t, x_{0}\right) v_{i}\right\|_{2}\right)
$$

i.e. $\left\|Y\left(t, x_{0}\right) v_{i}\right\| \sim \exp \left(\lambda_{i} t\right)$ as $t \rightarrow \infty$, or generally by $\lambda_{i}:=\limsup _{t \rightarrow \infty} \frac{1}{t} \log \left(\left\|Y\left(t, x_{0}\right) v_{i}\right\|_{2}\right)$ in case that the limit in (5) does not exist, where $v_{i} \in \mathbb{R}^{n} \backslash\{0\}$ are certain vectors which lead to all possible values $\lambda_{i}$ (when counted with multiplicities, $i=1, \ldots, n)$. More precisely, the functional

$$
\lambda^{+}(v):=\limsup _{t \rightarrow \infty} \frac{1}{t} \log \left(\left\|Y\left(t, x_{0}\right) v\right\|_{2}\right)
$$

has the properties

1. $\lambda^{+}(\alpha v)=\lambda(v)$ for every $v \in \mathbb{R}^{n}, \alpha \neq 0$,

2. $\lambda^{+}(v+w)=\max (\lambda(v), \lambda(w))$ for every $v, w \in \mathbb{R}^{n}$,

3. $\lambda^{+}(0)=-\infty$,

and due to these properties $\lambda^{+}$attains at most $n$ values $>-\infty$ on a filtration of linear subspaces $\{0\} \subset \operatorname{span}\left(v_{1}\right) \subset \cdots \subset$ $\operatorname{span}\left(v_{1}, \ldots, v_{n-1}\right) \subset \operatorname{span}\left(v_{1}, \ldots, v_{n}\right)=\mathbb{R}^{n}$ given by a normed ordered basis $v_{1}, \ldots, v_{n}$ of $\mathbb{R}^{n}$, i.e. $\lambda^{+}(v)$ is equal to a constant value $\lambda_{i}$ for $v=\sum_{j=1}^{i} \alpha_{j} v_{j}$ with $\alpha_{i} \neq 0$, $i=1, \ldots, n$ (for a proof see [3, Section 2]). We order the global Lyapunov exponents so that $\lambda_{1} \geq \cdots \geq \lambda_{n}$.

Eventually, global Lyapunov exponents to different initial states $x_{0}$ with same $\omega$-limit set $\omega\left(x_{0}\right)$ are identical, i.e. the global Lyapunov exponents do not change when the initial value is varied inside the basin of attraction of $\omega\left(x_{0}\right)$. However, a system with more than one $\omega$-limit set can have different global Lyapunov exponents.

Remark II.1 There are some obvious questions related to Lyapunov exponents:
1. Under which conditions is the limit superior in (6) a limit as in (5)?

In this case, the Lyapunov exponent $\lambda^{+}$is called exact.

2. Can the sum $\sum_{i=1}^{m} \lambda_{i}$ of Lyapunov exponents be viewed as long time growth or decay rates of the $m$-th dimensional volume spanned by the vectors $v_{1}, \ldots, v_{m}$ and transported along the orbit $x(t)$ ?

In this case, the Lyapunov exponent $\lambda^{+}$is called forward regular (for equivalent properties defining forward regularity see [3, Theorem 3.1]), and consequently forward regular systems are exact.

3. Does a negative largest Lyapunov exponent $\lambda_{1}<0$ imply asymptotic and exponential stability of $x(t)$ ? Generally not (see the counterexample of Perron discussed in [3, Example 1.1]), but in case of forward regularity yes due to Lyapunov's stability theorem [3, Theorem 1.2].

4. Does a distinguished normed ordered basis $v_{1}, \ldots, v_{n}$ of the filtration associated with (6) exist?

If not only the forward Lyapunov exponent $\lambda^{+}$, but also the backward Lyapunov exponent $\lambda^{-}(v):=$ $\lim \sup _{t \rightarrow-\infty} \frac{1}{|t|} \log \left(\left\|Y\left(t, x_{0}\right) v\right\|_{2}\right)$ satisfies the regularity assumption mentioned in 2. and the filtrations of both comply [3, Section 5], then the Lyapunov exponents are called regular and at each initial value $x_{0}$ with the same $\omega$-limit set there exists a distribution of linear subspaces $E_{\lambda_{i}}\left(x_{0}\right)$ which is invariant under $Y\left(t, x_{0}\right)$ and such that $v_{i} \in E_{\lambda_{i}}\left(x_{0}\right)$ implies (5). Vectors $v_{i} \in E_{\lambda_{i}}\left(x_{0}\right)$ are called covariant Lyapunov vectors to the Lyapunov exponent $\lambda_{i}$ at $x_{0}$. An algorithm to calculate these vectors has been proposed in [8], and the distribution $E$ is called Oseledets decomposition.

\section{4. Local Lyapunov exponents}

Using normed covariant Lyapunov vectors $v_{i}$, i.e. $v_{i} \in$ $E_{\lambda_{i}}\left(x_{0}\right)$ with $\left\|v_{i}\right\|_{2}=1$, as an $Y\left(t, x_{0}\right)$-invariant replacement for the eigenvectors $y_{i}$ to the eigenvalues $\mu_{i}\left(x_{0}\right)$ of the symmetric Jacobian, covariant local Lyapunov exponents can be defined by

$$
\lambda_{i}\left(x_{0}\right)=\lim _{t \rightarrow 0} \frac{1}{t} \ln \left(\left\|Y\left(t, x_{0}\right) v_{i}\right\|_{2}\right)=\left\langle D f\left(x_{0}\right)_{\mathrm{sym}} v_{i}, v_{i}\right\rangle .
$$

However, as the covariant Lyapunov vectors are difficult to determine, let us consider a method to calculate local Lyapunov exponents directly. The so called QR-method is based on a decomposition $Y\left(t, x_{0}\right)=Q(t) R(t)$ of $Y\left(t, x_{0}\right)$ into orthogonal matrices $Q(t)$ and right triangular matrices $R(t)$ with positive diagonal. For simplicity, denote $A(t):=D f(x(t))$, and let us assume for a moment that we already have $Y\left(t, x_{0}\right)=Q(t) R(t)$, then from

$$
\dot{Q} R+Q \dot{R}=\dot{Y}=A(t) Y=A(t) Q R
$$


we can read off $\dot{Q}=A(t) Q-Q U(t)$ with $U(t)$ such that $\dot{R}=U(t) R$. However, we need that $Q(t)$ is orthogonal, or equivalently that its time derivative satisfies $\dot{Q}=Q S$ with skew-symmetric matrices $S(t)$, as only in this case

$$
\begin{aligned}
\frac{d}{d t} Q^{T} Q & =\dot{Q}^{T} Q+Q^{T} \dot{Q}= \\
& =S^{T} Q^{T} Q+Q^{T} Q S=-S+S=0 .
\end{aligned}
$$

Therefore, we define $Q \mapsto S(Q, t)$ as the mapping which assigns to a matrix $Q$ the skew-symmetric matrix with the same strictly lower triangular part as $Q^{T} A(t) Q$, and we put $U(Q, t):=Q^{T} A(t) Q-S(Q, t)$, which consequently is an upper triangular matrix. Then the solution $Q(t)$ of the matrix ODE $\dot{Q}(t)=Q(t) S(Q(t), t)$, which is identical with $\dot{Q}(t)=A(t) Q(t)-Q(t) U(Q(t), t)$ by definition of $S(Q, t)$ and $U(Q, t)$, to the initial value $Q(0)=\mathrm{Id}$ is itself orthogonal for every $t$. On the other hand, having obtained this solution $Q(t)$ we can solve $\dot{R}(t)=U(Q(t), t) R(t)$ for the initial value $R(0)=$ Id to obtain time-dependent right triangular matrices $R(t)$ with positive diagonal such that $Y(t)=Q(t) R(t)$.

The diagonal elements of $U(t)=U(Q(t), t)$ are called QR local Lyapunov exponents $\lambda_{i}(x(t))$ along the orbit and evolve in time so that

$$
\lambda_{i}=\lim _{t \rightarrow \infty} \frac{1}{t} \int_{0}^{t} \lambda_{i}(x(s)) d s .
$$

Note that for a calculation of the local Lyapunov exponents it is not necessary to solve the equation $\dot{R}(t)=$ $U(t) R(t)$, In the special case of conservative systems, where trace $(A(t))=0$ and consequently $Y(t)$ preserves the standard volume form on $\mathbb{R}^{2 n}$, the equation trace $(R(t))=0$ holds, i.e. the general local Lyapunov exponents $\lambda_{i}(x(t))$ and therefore also the global Lyapunov exponents add up to 0 .

\section{5. Linear Poisson manifolds}

To define Hamiltonian systems, let us consider the simplest case of a Poisson manifold $M$, namely the linear space $M:=\mathbb{R}^{N}, N \in \mathbb{N}$, endowed with a linear Poisson structure given by a (constant) skew-symmetric matrix $J$ (for the general case, see [10]).

Definition II.2 A real square matrix $J$ is called skewsymmetric, if $J^{T}=-J$.

\section{Example II.3}

1. The matrix $J=\left(\begin{array}{cc}0 & \mathrm{Id}_{n} \\ -\mathrm{Id}_{n} & 0\end{array}\right)$ on the evendimensional linear space $\mathbb{R}^{2 n}, n \in \mathbb{N}$, is not only skewsymmetric but even invertible. Therefore, $J$ is called a linear symplectic structure, and $\mathbb{R}^{2 n}$ endowed with this matrix $J$ is called the standard symplectic linear space. Note that this matrix $J$ satisfies $J^{-1}=-J$ or equivalently $J^{2}=-\mathrm{Id}_{2 n}$, thus $J$ may even be viewed as a complex structure on $\mathbb{R}^{2 n}$.
2. The matrix $J=\left(\begin{array}{ccc}0 & \mathrm{Id}_{n} & 0 \\ -\mathrm{Id}_{n} & 0 & 0 \\ 0 & 0 & 0_{k}\end{array}\right)$ on the (possibly odd-dimensional) linear space $M:=\mathbb{R}^{2 n+k}, n, k \in \mathbb{N}$, is skew-symmetric and thus a linear Poisson structure, but not invertible and therefore not a linear symplectic structure.

On a linear Poisson manifold $(M, J)$, the Hamiltonian ODE associated with a Hamiltonian function $H: M \rightarrow \mathbb{R}$ is the autonomous ODE

$$
\dot{x}(t)=J(\nabla H)(x(t))
$$

where $f(x):=J(\nabla H)(x)$ is called the Hamiltonian vector field to $H$, and $H$ is preserved along orbits due to $\frac{d}{d t} H(x(t))=(\nabla H)(x(t))^{T} J(\nabla H)(x(t))=0$.

Definition II.4 A real square matrix $A$ (which in this context should be interpreted as a linear vector field $x \mapsto A x$ ) is called Hamiltonian (or infinitesimally Poisson) w.r.t. the skew-symmetric matrix $J$, iff $A J+J A^{T}=0$.

For every linear Hamiltonian vector field $A$, if $\lambda$ is an eigenvalue of $A$, then also $\bar{\lambda},-\lambda$ and $-\bar{\lambda}$ are eigenvalues of $A$. In fact, the eigenvalues of $A$ and $A^{T}$ are identical due to $\operatorname{det}(A-\lambda \mathrm{Id})=\operatorname{det}\left(A^{T}-\lambda \mathrm{Id}\right)$, and if $u$ is an eigenvector of $A^{T}$ to the eigenvalue $\lambda$, i.e. $A^{T} u=\lambda u$, then $A J u=-J A^{T} u=-\lambda J u$, i.e. $J u$ is an eigenvector to the eigenvalue $-\lambda$ of $A$. Thus, with $\lambda$ also $-\lambda$ is an eigenvalue of $A$. Moreover, as $A$ is a real matrix, with $\lambda$ also $\bar{\lambda}$ is an eigenvalue.

\section{CHARACTERISTIC EXPONENTS IN HAMILTONIAN SYSTEMS}

\section{1. Eigenvalues of the symmetric Jacobian occur in pairs}

The matrices $A(t):=J(\operatorname{Hess} H)(x(t))$ determining the time evolution of the linearization $\dot{y}=J(\operatorname{Hess} H)(x(t)) y$ of the Hamiltonian system (9) along an orbit $x(t)$ are timedependent linear Hamiltonian vector fields due to

$$
\begin{aligned}
A(t) J+J A(t)^{T} & =J(\operatorname{Hess} H)(x(t)) J \\
& +J(\operatorname{Hess} H)(x(t))^{T} J^{T}=0,
\end{aligned}
$$

where we used symmetry $(\operatorname{Hess} H)(x(t))^{T}=(\operatorname{Hess} H)$ $(x(t))$ and skew-symmetry $J^{T}=-J$. Particularly, for the Hamiltonian vector field $f(x):=J(\nabla H)(x)$ on the right hand side of (9) the (ordered) eigenvalues $\mu_{i}\left(x_{0}\right)$ of the symmetric Jacobian $D f\left(x_{0}\right)_{\text {sym }}=A(0)_{\text {sym }}$ considered in section II. 2 occur at every point $x_{0}$ in pairs $\mu_{i}\left(x_{0}\right)=$ $-\mu_{n+1-i}\left(x_{0}\right), i=1, \ldots, n$. 


\section{2. Symplectic / SVD local Lyapunov exponents occur in pairs}

Regarding the global and local Lyapunov exponents considered in sections II. 3 and II. 4, for Hamiltonian systems on linear symplectic manifolds [2] shows that global Lyapunov exponents occur in pairs $(\lambda,-\lambda)$. Let us give here a new proof of this fact by proving the same for local Lyapunov exponents defined via an adopted decomposition of $Y(t, x)$.

Note that the orthogonal matrices $Q(t)$ of the QRdecomposition of $Y\left(t, x_{0}\right)$ generally do not preserve the Poisson structure $J$, which makes a proof of the pairing property impossible. Therefore, we use the slightly different, but more appropriate Iwasawa decomposition in the Lie group $S p(2 n, \mathbb{R})$ of linear real symplectic transformations. Note that if $\dot{Y}=A(t) Y$ with the time-de- pendent linear Hamiltonian vector fields $A(t)$, then $\frac{d}{d t} Y^{-1}=-Y^{-1} A(t)$ and thus $\frac{d}{d t}\left(Y(t)^{-1} J\left(Y(t)^{-1}\right)^{T}\right)=$ $-Y(t)^{-1}\left(A(t) J+J A(t)^{T}\right)\left(Y(t)^{-1}\right)^{T}=0$ is valid, i.e. $Y(t)^{-1}$ preserves the linear Poisson structure $J$ and is therefore a linear real symplectic transformation provided that $J$ is invertible and hence symplectic.

The Iwasawa decomposition of a linear real symplectic transformations is computed in [4] via the QR decomposition, see [12] for general Lie groups. It reads as $Y(t)=$ $K(t) A N(t)$ with an orthogonal matrix $K(t)$ preserving $J$, and a matrix $A N(t)$ of the form $\left(\begin{array}{cc}A N_{1} & A N_{2} \\ 0 & A N_{1}^{-T}\end{array}\right)$ with upper triangular $A N_{1}$, i.e. the matrix $A N(t)$ is not an upper triangular matrix, but rather similar to such a matrix. The use of the Iwasawa decomposition for the computation of Lyapunov exponents has been proposed in [6], however, there
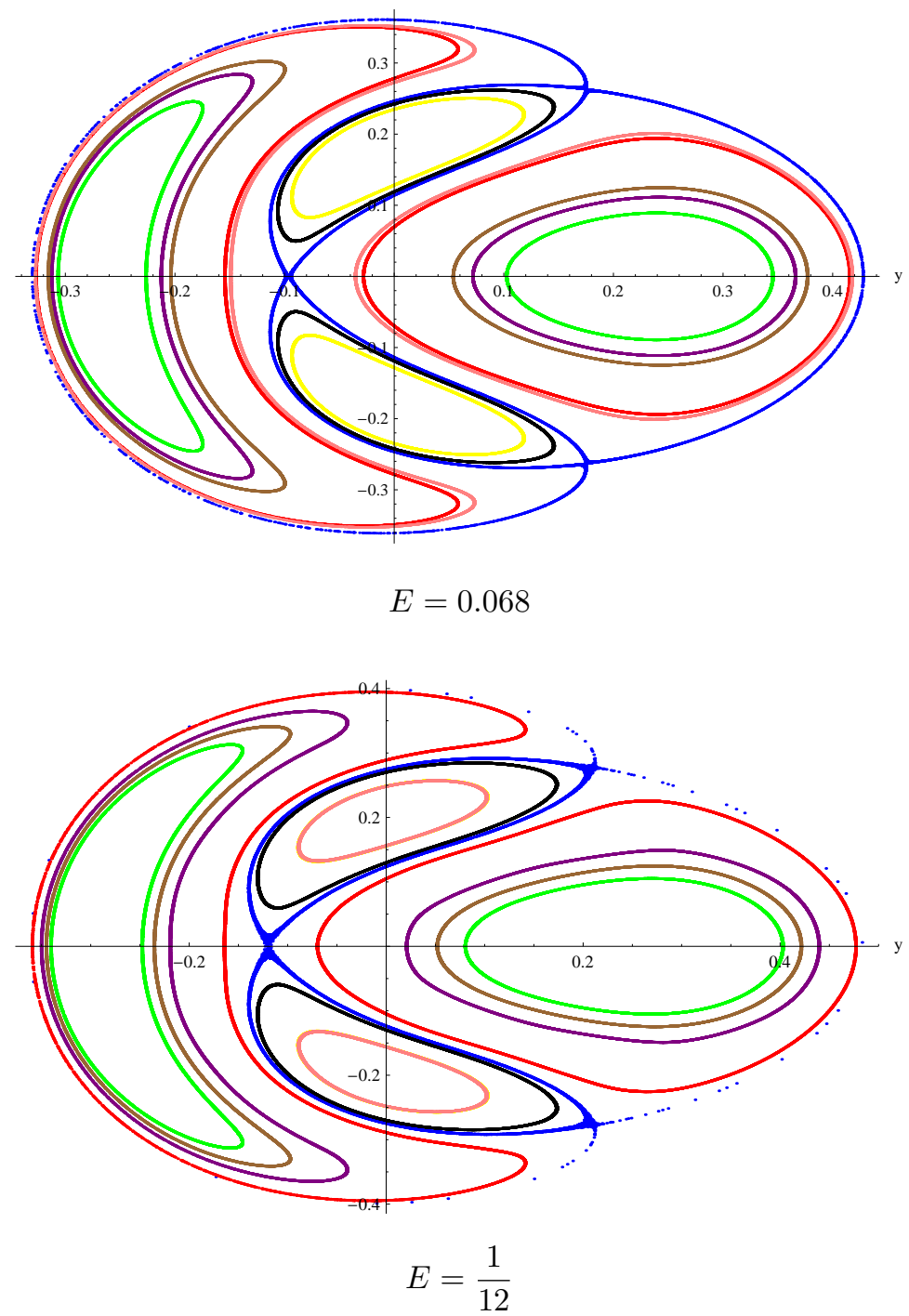

Fig. 1. Poincaré sections of the Hénon-Heiles system in the $\left(y, p_{y}\right)$-plane 


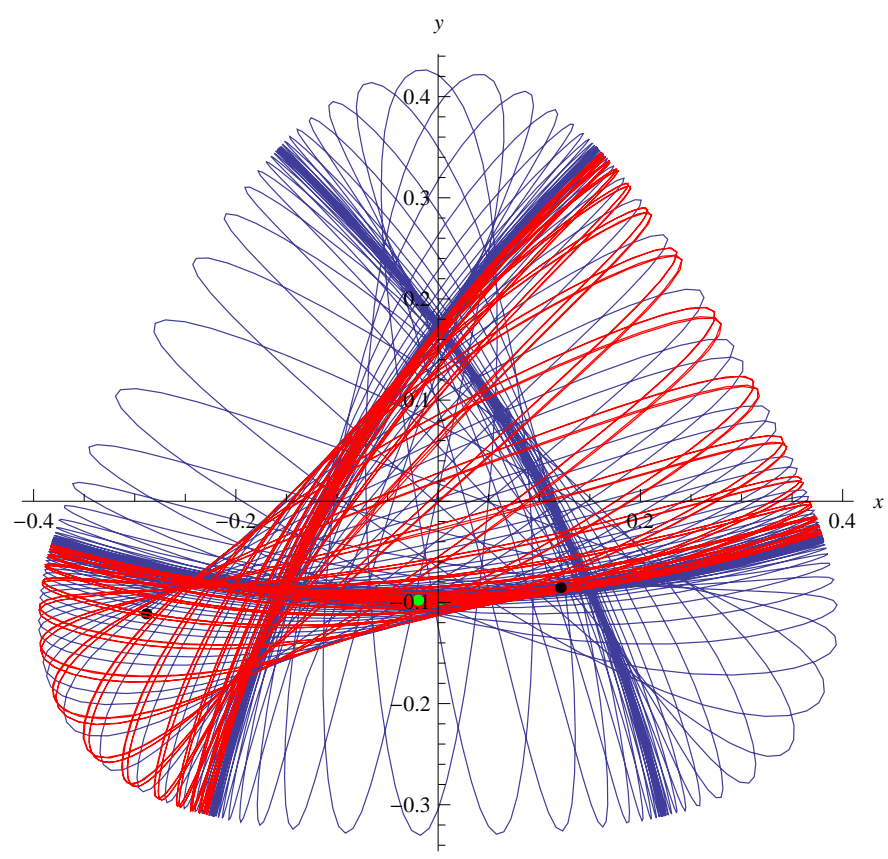

Fig. 2. Plot of the blue trajectory (initial values: $x_{0}=0 ; y_{0}=-0.09772 ; p_{x, 0}=0.354723 ; p_{y, 0}=0 ; E=0.068$ ) and a red trajectory that starts very close to it after 500 steps. They start spreading off after about 50 steps. Therefore the system seems to be chaotic

$A N$ is assumed to be an upper triangular matrix which is not true in general.

For the Iwasawa decomposition $Y(t)=K(t) A N(t)$ the matrices $A N(t)^{-1}$ preserve $J=K(t)^{T} J K(t)$, and particularly the matrix $U(t)$ with $A N(t)=U(t) A N(t)$ is a linear Hamiltonian vector field w.r.t. $J$ of the form $U(t)=\left(\begin{array}{cc}U_{1} & U_{2} \\ 0 & -U_{1}^{T}\end{array}\right)$ with upper triangular $U_{1}$. Now the diagonal elements of $U$ are the symplectic local Lyapunov exponents along the orbit, and because the diagonal elements of $U_{1}$ have a sign complementary to the diagonal elements of $-U_{1}^{T}$, the (ordered) local Lyapunov exponents occur in pairs $\lambda_{i}(x(t))=-\lambda_{n+1-i}(x(t))$, Finally, from this pairing property of the local Lyapunov exponents, the fact that by (8) the global Lyapunov exponents are time averages of the local Lyapunov exponents, and by calling averages $\frac{1}{t} \int_{0}^{t} \lambda_{i}(x(s)) d s$ for $0<t<+\infty$ the finite time Lyapunov exponents, we deduce the following theorem.

Theorem III.1 For a Hamiltonian system on a linear symplectic manifold, if the Lyapunov exponents are regular, then the symplectic local Lyapunov exponents occur in pairs $(\lambda,-\lambda)$, and the same holds for the symplectic finite time and the global Lyapunov exponents.

While the Iwasawa decomposition above is adapted to the symplectic group and therefore provides local Lyapunov exponents with the pairing property, the SVD method automatically gives local Lyapunov exponents with the pairing property in the case of a complex structure, i.e. in the case where $J$ is both skew-symmetric and orthogonal.
In fact, if $Y(t)=U(t) S(t) V(t)^{T}$ is the SVD decomposition, then $S^{\prime}=\operatorname{diag}\left(U^{T} Y^{\prime} V\right)=\operatorname{diag}\left(U^{T} A U\right) U^{T} Y V=$ $\operatorname{diag}\left(U^{T} A U\right) S$. Because $Y$ preserves $J$, i.e. $Y J Y^{T}=J$ and in the case of a complex structure also $Y^{T} J Y=$ $J$, even $Y Y^{T}$ preserves $J$ due to $J=Y J Y^{T}=$ $Y Y^{T} J Y Y^{T}$ and symmetry of $Y Y^{T}$. Therefore, if $u$ is eigenvector of $Y Y^{T}$ to the eigenvalue $\sigma^{2}$, then $J u=$ $Y Y^{T} J Y Y^{T} u=\sigma^{2} Y Y^{T} J u$, i.e. also $J u$ is an eigenvector to $\sigma^{2}$, and $J u$ is orthogonal to $u$ due to $u^{T} J u=0$. Hence, we have $S^{2}=\operatorname{diag}\left(\sigma_{1}^{2}, \ldots, \sigma_{n}^{2}, \sigma_{1}^{2}, \ldots, \sigma_{n}^{2}\right)$ and $U=\left(u_{1}, \ldots, u_{n}, J u_{1}, \ldots, J u_{n}\right)$. Finally, $U^{T} J U=$ $\left(u_{1}, \ldots, u_{n}, J u_{1}, \ldots, J u_{n}\right)^{T}\left(J u_{1}, \ldots, J u_{n},-u_{1}, \ldots,-u_{n}\right)=$ $\left(\begin{array}{cc}0 & -\mathrm{Id} \\ \mathrm{Id} & 0\end{array}\right)=J$ and hence $J U=U J$. Due to this equation we have $U^{T} A U J+J\left(U^{T} A U\right)^{T}=U^{T}\left(A J+J A^{T}\right) U=0$. Therefore, $U^{T} A U$ is infinitesimally Poisson, and thus for $\lambda$ on the diagonal of $U^{T} A U$ also $-\lambda$ occurs on the diagonal. This shows that the SVD local Lyapunov exponents given by the diagonal of $U^{T} A U$ occur in pairs $(\lambda,-\lambda)$.

Theorem III.2 For a Hamiltonian system on the standard symplectic manifold $\mathbb{R}^{2 n}$, if the Lyapunov exponents are regular, then the SVD local Lyapunov exponents occur in pairs $(\lambda,-\lambda)$, and the same holds for the SVD finite time and the global Lyapunov exponents.

Finally, the covariant Lyapunov exponents are in some aspects related to the SVD local Lyapunov exponents. In fact, the columns of $\lim _{t \rightarrow \infty} U(t)$ are the eigenvectors of $\lim _{t \rightarrow \infty}\left(Y(t) Y(t)^{T}\right)^{1 / 2 t}$ and $\lim _{t \rightarrow \infty} V(t)$ consists of the eigen- 
vectors of $\lim _{t \rightarrow \infty}\left(Y(t)^{-1} Y(t)^{-T}\right)^{1 / 2 t}$, and the two orthogonal decompositions given by the columns of $U(t)$ resp. $V(t)$ interpolate between these eigenvectors for $-\infty<t<\infty$, while the SVD local Lyapunov exponents interpolate between the forward resp. backward global Lyapunov exponents. In this aspect they are similar to Oseldets decomposition and the covariant local Lyapunov exponents, however, Oseldets decomposition is in general non-orthogonal while the columns of $U(t)$ resp. $V(t)$ are orthogonal.

\section{LOW-DIMENSIONAL NUMERICAL EXAMPLES}

\section{1. The Hénon-Heiles system}

The Hamiltonian system on the standard symplectic linear space $\mathbb{R}^{4}$ with Hamiltonian

$H\left(x, y, p_{x}, p_{y}\right):=\frac{1}{2}\left(p_{x}^{2}+p_{y}^{2}\right)+\frac{1}{2}\left(x^{2}+y^{2}\right)+x^{2} y-\frac{1}{3} y^{3}$

is called the classical Hénon-Heiles system. It is nonintegrable [14] and reads in coordinates as

$$
\begin{aligned}
\dot{x} & =p_{x} \\
\dot{y} & =p_{y} \\
\dot{p}_{x} & =-x-2 x y \\
\dot{p}_{y} & =-y-x^{2}+y^{2}
\end{aligned}
$$

Denote by $E(x, y):=\frac{1}{2}\left(x^{2}+y^{2}\right)+x^{2} y-\frac{1}{3} y^{3}$ the potential energy of the Hénon-Heiles system. Then solution curves with no initial momentum (i.e. $p_{x}(0)=0=p_{y}(0)$ ) can not escape the region $\left\{(x, y) \mid E(x, y) \leq \frac{1}{6}\right\}$ in the $(x, y)$-plane, which due to

$$
E(x, y)-\frac{1}{6}=\frac{1}{6}(\sqrt{3} x+y-1)(\sqrt{3} x-y+1)(2 y+1)
$$

is a closed triangle. The value $E=\frac{1}{6}$ is the largest one where escape does not occur, therefore we restrict our attention to solutions starting in this closed triangle with no initial momentum and to values $E \leq \frac{1}{6}$.

Note the symmetry that for a solution $\left(x, y, p_{x}, p_{y}\right)$ also $\left(-x, y,-p_{x}, p_{y}\right)$ is a solution. Due to this symmetry, the plane $x=0$ seems to be a good choice for plotting Poincaré sections. At each energy level $0<H<\frac{1}{6}$, the only solution entirely inside this plane is a periodic orbit implicitly given by $\frac{1}{2} p_{y}^{2}+\frac{1}{2} y^{2}-\frac{1}{3} y^{3}=H$. Further, as $p_{x}$ is determined by the energy level, we plot the coordinates $\left(y, p_{y}\right)$ when an orbit intersects the plane $x=0$.

These Poincaré sections seem to indicate that the system is already chaotic at energy $E=\frac{1}{12}$ (and even for a little smaller value like $E \approx 0.068$ ), see also [15]: The system (11) has four equilibrium points at momenta $\left(p_{x}, p_{y}\right)=(0,0)$ and positions $(x, y)=(0,0)$ (with energy $H=0),(x, y)=(0,1)$ and $(x, y)=\left( \pm \frac{\sqrt{3}}{2},-\frac{1}{2}\right)$ (each with energy $H=\frac{1}{6}$ ), all lie in the triangle $\left\{(x, y) \mid E(x, y) \leq \frac{1}{6}\right\}$. For small energies, these stable equilibria are surrounded by invariant tori. However, for $E \approx 0.068$ a chaotic sea appears near an unstable periodic orbit through $\left(x, y, p_{y}\right) \approx(0,-0.097,0)$. The following trajectory plot provides numerical evidence for chaos, although the chaotic sea can only be seen by zooming in near to this point.

At energy $E=\frac{1}{12}$, a thick chaotic sea can already be seen in the Poincaré section near this point by eye without zooming in. As the energy level increases, the chaotic sea becomes larger. At $H=\frac{1}{6}$ there are just a few non-chaotic parts.

Lyapunov exponents in the Hénon-Heiles system have been calculated by [13]. Figures 3 to 5 show the QR, symplectic and SVD local Lyapunov exponents $\lambda_{2}$ and $\lambda_{3}$ for two orbits, a chaotic orbit with global Lyapunov exponents $\approx(0.127,0,0,-0.127)$ and a periodic orbit. In both cases, the local Lyapunov exponents calculated by the QR method are not symmetric, while the symplectic and SVD local Lyapunov exponents have the pairing property.

\section{2. Sixth order generalization of the Hénon-Heiles system}

Generalizations of the Hénon-Heiles system have been studied by Zachilas, particularly polynomial truncations of even order [16]. The Hamiltonian of the sixth order approximation of the Hénon-Heiles system is

$$
\begin{aligned}
& H_{6}\left(x, y, p_{x}, p_{y}\right):=\frac{1}{2}\left(p_{x}^{2}+p_{y}^{2}\right)+\frac{1}{2}\left(x^{2}+y^{2}\right)+x^{2} y \\
- & \frac{1}{3} y^{3}+\frac{1}{2} x^{4}+x^{2} y^{2}+\frac{1}{2} y^{4}+x^{4} y+\frac{2}{3} x^{2} y^{3}-\frac{1}{3} y^{5} \\
+ & \frac{1}{5} x^{6}+x^{4} y^{2}+\frac{1}{3} x^{2} y^{4}+\frac{11}{45} y^{6} .
\end{aligned}
$$

The corresponding Hamiltonian system reads as

$$
\begin{aligned}
\dot{x} & =p_{x} \\
\dot{y} & =p_{y} \\
\dot{p}_{x} & =-x-2 x y-2 x^{3}-2 x y^{2}-4 x^{3} y \\
& -\frac{4}{3} x y^{3}-\frac{6}{5} x^{5}-4 x^{3} y^{2}-\frac{2}{3} x y^{4} \\
\dot{p}_{y} & =-y-x^{2}+y^{2}-2 x^{2} y-2 y^{3} \\
& -x^{4}-2 x^{2} y^{2}+\frac{5}{3} y^{4}-2 x^{4} y-\frac{4}{3} x^{2} y^{3}-\frac{22}{15} y^{5} .
\end{aligned}
$$

Let

$$
\begin{aligned}
E(x, y):= & \frac{1}{2}\left(x^{2}+y^{2}\right)+x^{2} y-\frac{1}{3} y^{3}+\frac{1}{2} x^{4}+x^{2} y^{2} \\
& +\frac{1}{2} y^{4}+x^{4} y+\frac{2}{3} x^{2} y^{3}-\frac{1}{3} y^{5}+\frac{1}{5} x^{6}+x^{4} y^{2} \\
& +\frac{1}{3} x^{2} y^{4}+\frac{11}{45} y^{6}
\end{aligned}
$$

denote the potential energy of $H_{6}$. In contrast to the HénonHiles system there is no escape energy in $H_{6}$ due to the 

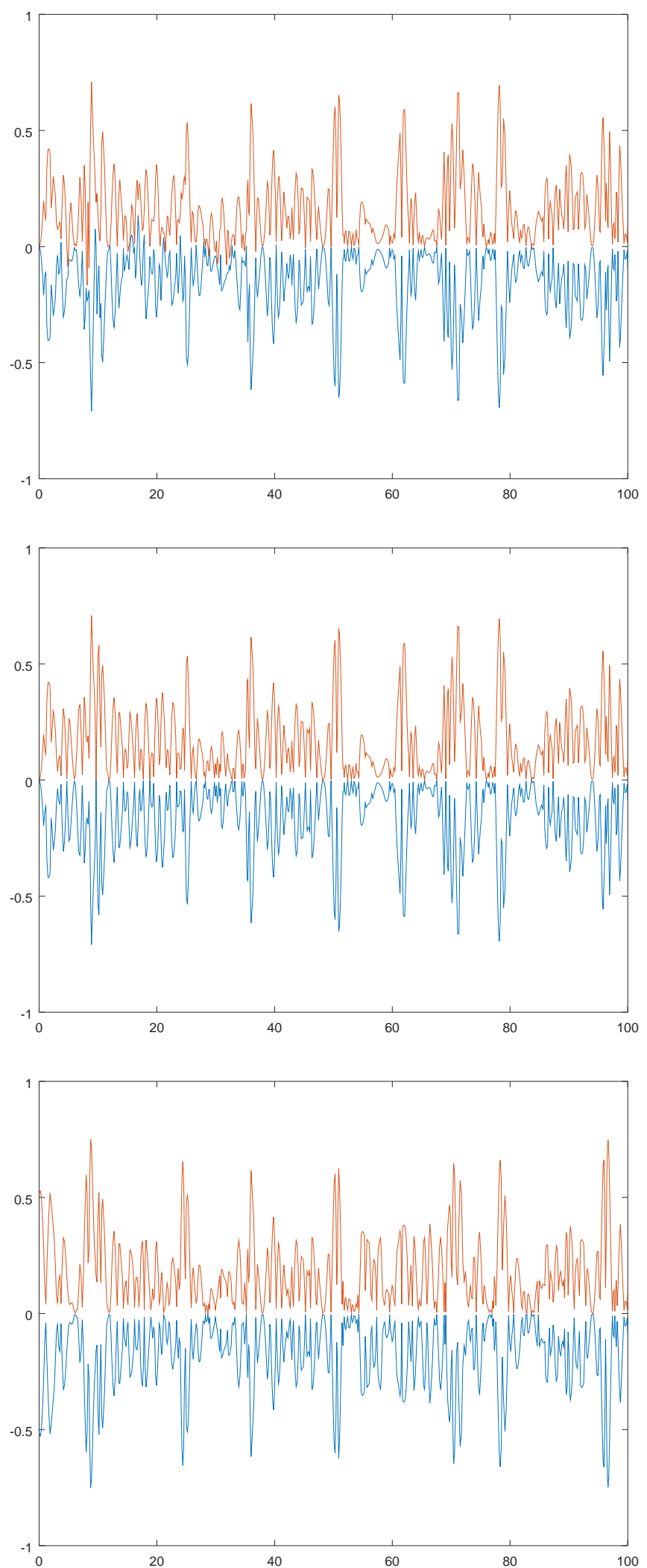

Fig. 3. For the chaotic trajectory with initial values $x_{0}=0 ; y_{0}=\frac{1}{2} ; p_{x, 0}=\frac{1}{\sqrt{12}} ; p_{y, 0}=\frac{1}{\sqrt{12}} ; H=\frac{1}{6}$; the (ordered) QR local Lyapunov exponents $\lambda_{2}, \lambda_{3}$ are not symmetric up to time $\approx 40$, while the symplectic and SVD local Lyapunov exponents are symmetric 

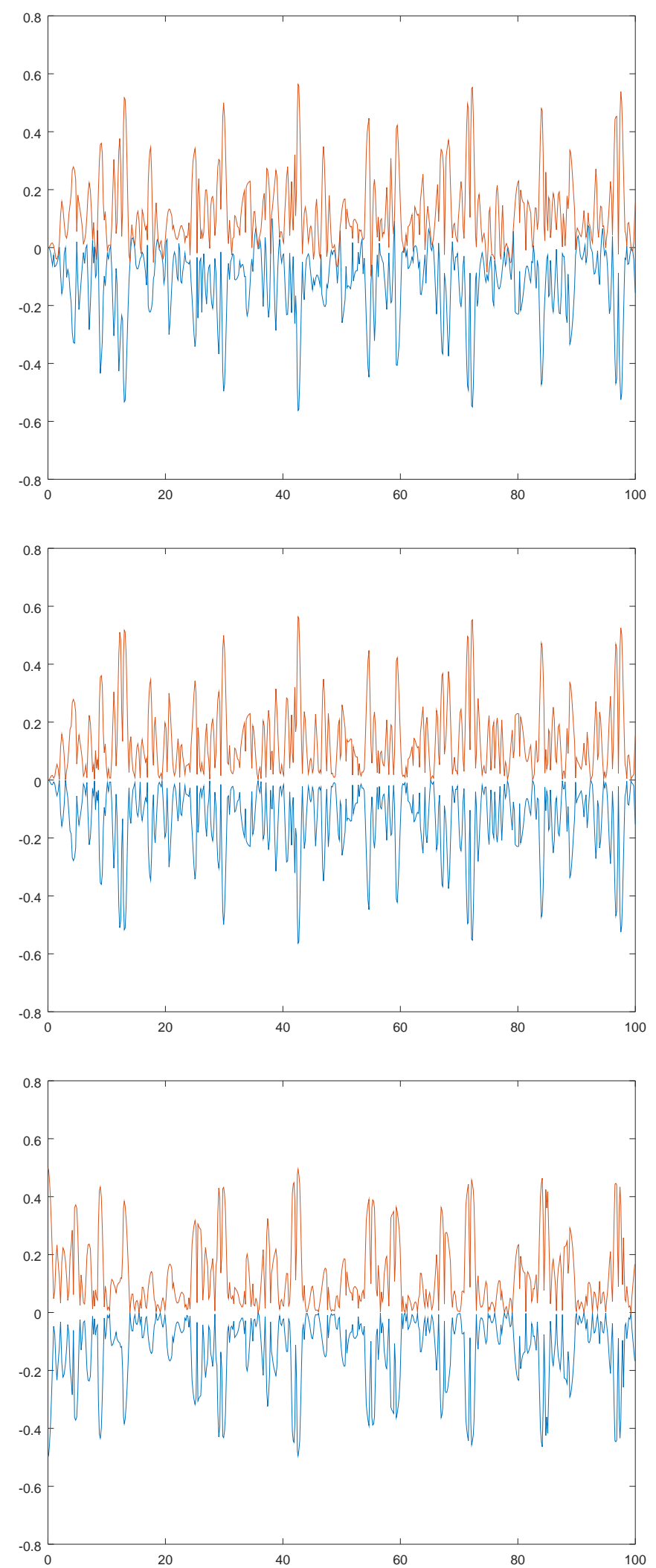

Fig. 4. For the periodic trajectory with initial values $x_{0}=0 ; y_{0}=\frac{1}{2} ; p_{x, 0}=\frac{1}{\sqrt{6}} ; p_{y, 0}=0 ; H=\frac{1}{6}$; the (ordered) QR local Lyapunov exponents $\lambda_{2}, \lambda_{3}$ are not symmetric the whole time, while the symplectic and SVD local Lyapunov exponents are symmetric 

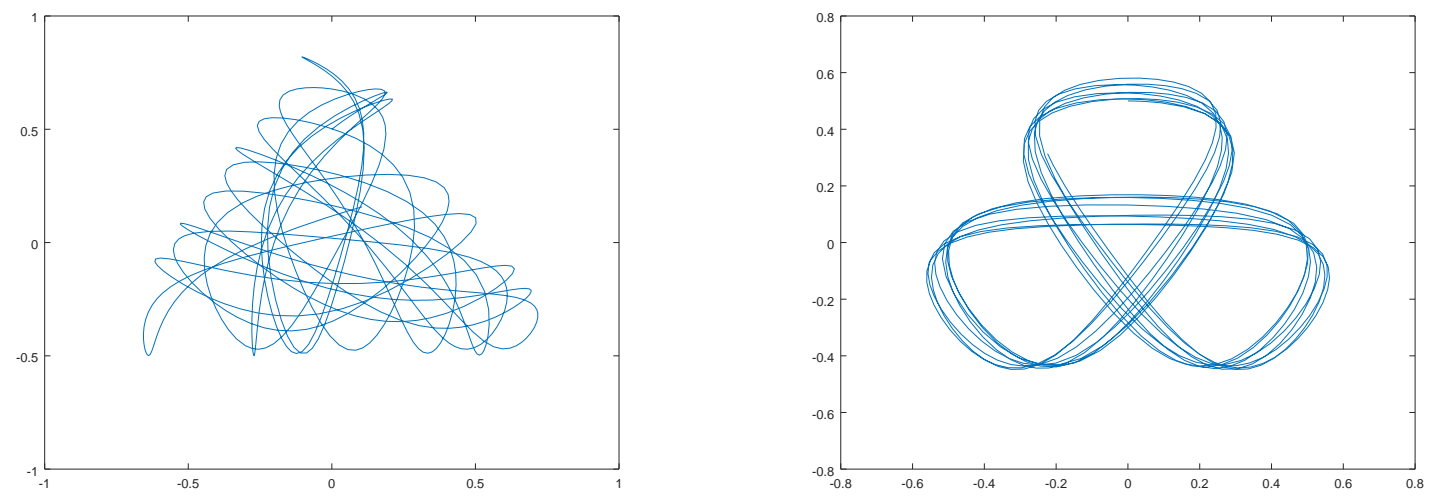

Fig. 5. The chaotic and periodic trajectory of Figures 3 resp. 4 in the $(x, y)$-plane

highest order terms $\frac{1}{5} x^{6}+x^{4} y^{2}+\frac{1}{3} x^{2} y^{4}+\frac{11}{45} y^{6}$ of degree 6 in the potential energy. Analogously to the Hénon-Heiles system, we choose again the plane $x=0$ for plotting Poincaré sections, since there is again the symmetry that for a solution $\left(x, y, p_{x}, p_{y}\right)$ also $\left(-x, y,-p_{x}, p_{y}\right)$ is a solution of $H_{6}$ due to the fact that $E(x, y)$ contains only items in which $x$ occurs of even order.

Now we focus on total energy $H_{6}=18$, because there seem to exist two separated chaotic regions / islands (more precisely: ergodic components) for this particular energy. The numerical evaluations in Figures 6 and 7 seem to manifest there exist two separated chaotic regions. We can also see in Figures 8 and 9 that the Lyapunov spectrum is different for the above considered two trajectories taken from different chaotic regions.

Consequently, there is numerical evidence that the chaotic regions (I) and (II) are separated. Yet, there seems to be no proof in the literature that this system has two separated chaotic regions. A proof of the existence of distinct chaotic ergodic components seems to be known merely for mushroom billards, see [5]. In fact, mushroom billiards with arbitrary numbers of chaotic and regular ergodic components can be constructed.

\section{CONCLUSION}

In this article, we successfully proved the pairing property for symplectic and SVD local Lyapunov exponents, while showing that QR local Lyapunov exponents are in general not symmetric. Further, we gave an example of a simple Hamiltonian system with two separated chaotic seas. However, we were not able to rigorously prove the pairing property for covariant Lyapunov exponents (although our proof of symmetry for SVD local Lyapunov exponents indicates that this claim of [11] holds true), and we did not give a proof that there exist two chaotic seas in the sixth order generalization of the Hénon-Heiles system. Moreover, we think that in the Hamiltonian case it is not necessary to assume regularity, as regularity should hold automatically, see e.g. [6]. Yet, these questions seem to remain open problems.

\section{A. ON THE IWASAWA DECOMPOSITION}

For the readers convenience, in this appendix we give an introduction to the Iwasawa decomposition. For a more detailed mathematical exposition, see [6] for the idea to use the Iwasawa decomposition in the computation of Lyapunov exponents in Hamiltonian systems, and [4] for the computation of the Iwasawa decomposition of a symplectic matrix. Particularly, [4] includes an easily accessible MATLAB code to compute of the Iwasawa decomposition of symplectic matrices.

Mathematically, the main point about the Iwasawa decomposition of a Lie group $\mathcal{G}$ of matrices is that it decomposes the Lie group $\mathcal{G}=\mathcal{K} \mathcal{A N}$ into a compact part $\mathcal{K}$, an abelian part $\mathcal{A}$ and a nilpotent part $\mathcal{N}$. In this general sense, the Iwasawa decomposition exists for every classical Lie group of non-compact type, see [12].

In this article we concentrate on two concrete cases, the case of the general linear group $G L(n, \mathbb{R})$ of invertible $(n \times n)$-matrices, or equivalently the special linear group $S L(n, \mathbb{R})$ of matrices with determinant 1 (note that every invertible matrix can be decomposed into a non-zero multiple of a matrix with determinant 1 , which is why we consider the two groups as equivalent), and the case of the symplectic group

$$
S p(2 n, \mathbb{R}):=\left\{S \in S L(2 n, \mathbb{R}): S^{T} J S=J\right\}
$$

consisting of symplectic $(2 n \times 2 n)$-matrices $S$, where $J$ is given in Example 2.3 (i). Note that both $G L(n, \mathbb{R})$ resp. 


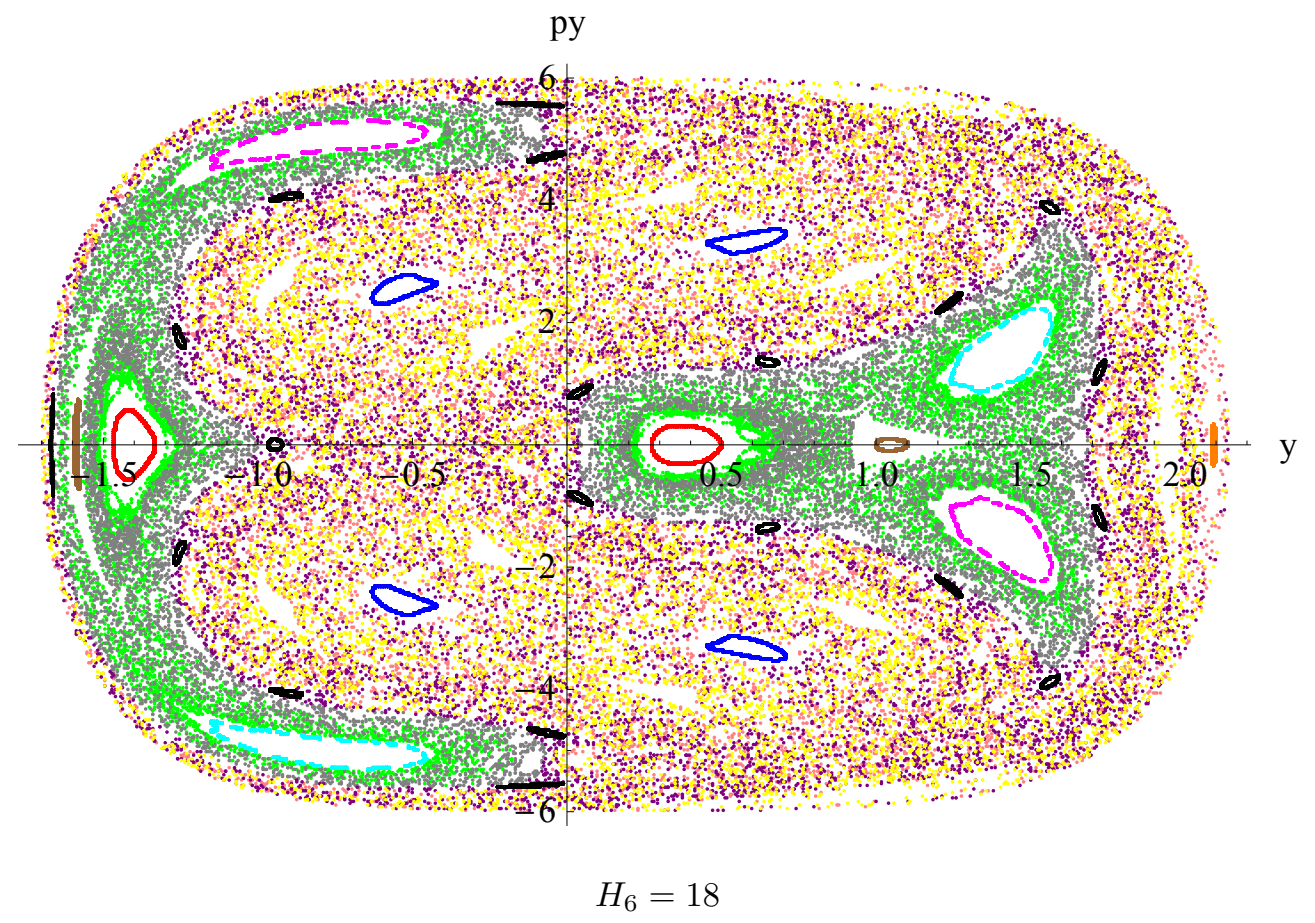

Fig. 6. Here we can see two separated chaotic seas - a big one (I) that is in the middle of the Poincaré section and a smaller one (II) that is on the left and on the right of the Poincare section. The initial values can be found in the following table:

\begin{tabular}{c|c|c|c|c}
$x$ & $p_{x}$ & $y$ & $p_{y}$ & Color \\
\hline \hline 0 & 6 & 0 & 0 & pink \\
\hline 0 & 5.982 & 0.5 & 0 & red \\
\hline 0 & 5.956 & 0.75 & 0 & green \\
\hline 0 & 5.903 & 1 & 0 & brown \\
\hline 0 & 5.673 & -1 & 0 & gray \\
\hline 0 & 3.373 & 2 & 0 & purple \\
\hline 0 & 4.703 & 1.8 & 0 & yellow \\
\hline 0 & 5.054 & 0.5 & -3.2 & blue \\
\hline 0 & 1.901 & 2.1 & 0 & orange \\
\hline 0 & 3.350 & -0.8 & 4.8 & magenta \\
\hline 0 & 3.350 & -0.8 & -4.8 & cyan \\
\hline 0 & 5.825 & 0.65 & 1.3 & black \\
\hline
\end{tabular}

$S L(n, \mathbb{R})$ and $S p(2 n, \mathbb{R})$ are Lie groups w.r.t. matrix multiplication.

For this article, the main point is that for the groups $G L(n, \mathbb{R})$ resp. $S L(n, \mathbb{R})$ the Iwasawa decomposition of a matrix $Y$ in these groups is identical with the usual QRdecomposition $Y=Q R$, i.e. the compact part (the group $O(n, \mathbb{R})$ of orthogonal matrices for $G L(n, \mathbb{R})$ resp. the special orthogonal group $S O(n, \mathbb{R})$ of orthogonal matrices with determinant 1 for $S L(n, \mathbb{R})$ ) of the matrix $Y$ in $G L(n, \mathbb{R})$ resp. $S L(n, \mathbb{R})$ is given by $Q$, while the abelian part (diagonal matrices with positive entries for $G L(n, \mathbb{R})$ resp. positive entries multiplying up to 1 in case of $S L(n, \mathbb{R})$ ) and the nilpotent part (upper triangular matrices with unit diagonal) are subsumed in the right triangular matrix $R$.
However, for the symplectic group $S p(2 n, \mathbb{R})$ the Iwasawa decomposition is different. There the compact part merely consists of those orthogonal $(2 n \times 2 n)$-matrices $K$ which are additionally symplectic, while the abelian part (diagonal matrices of the form $\operatorname{diag}\left(a_{1}, \ldots, a_{n}, \frac{1}{a_{1}}, \ldots, \frac{1}{a_{n}}\right)$ with positive entries) and the nilpotent part (matrices of the form $\left(\begin{array}{cc}N_{1} & N_{2} \\ 0 & N_{1}^{-T}\end{array}\right)$ with unit upper triangular $N_{1}$ satisfying $N_{1} N_{2}^{T}=N_{2} N_{1}^{T}$ ) can be subsumed into a matrix $A N$ of the form $A N:=\left(\begin{array}{cc}A N_{1} & A N_{2} \\ 0 & A N_{1}^{-T}\end{array}\right)$ with upper triangular $A N_{1}$, i.e. the matrix $A N$ is not an upper triangular matrix, but rather similar to such a matrix. In the main part of the article, we use this Iwasawa decomposition to show that the symplectic local 

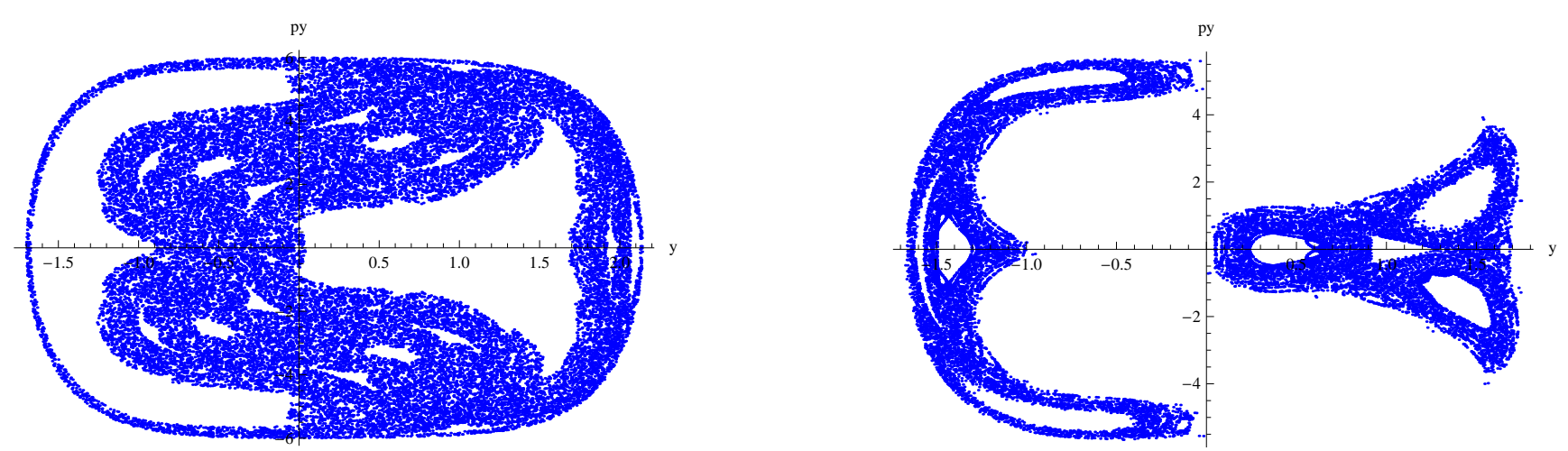

Fig. 7. Above we see the Poincaré sections of two trajectories starting in (I) at $x_{0}=0 ; y_{0}=0 ; p_{x, 0}=6 ; p_{y, 0}=0 ; H_{6}=18 ;$ and in (II) at $x_{0}=0 ; y_{0}=0.75 ; p_{x, 0}=5.9559634 ; p_{y, 0}=0 ; H_{6}=18$; calculated using Mathematica's NDSolve with default adapted step size

Lyapunov exponents are paired. For pedagogical purposes, in this appendix we provide explicit calculations for two simple systems.

\section{A. 1. A two-dimensional example}

$$
\text { For } \begin{aligned}
S= & \left(\begin{array}{ll}
a & b \\
c & d
\end{array}\right) \in S L(2, \mathbb{R}), \text { the identity } \\
S^{T} J S & =\left(\begin{array}{ll}
a & c \\
b & d
\end{array}\right)\left(\begin{array}{cc}
0 & 1 \\
-1 & 0
\end{array}\right)\left(\begin{array}{ll}
a & b \\
c & d
\end{array}\right)= \\
& =\left(\begin{array}{cc}
0 & a d-b c \\
b c-a d & 0
\end{array}\right)= \\
& =\left(\begin{array}{cc}
0 & 1 \\
-1 & 0
\end{array}\right)=J
\end{aligned}
$$

holds, i.e. $S L(2, \mathbb{R})=S p(2, \mathbb{R})$. Consider the following three subgroups of $\operatorname{Sp}(2, \mathbb{R})$ :

$$
\begin{aligned}
& \mathcal{K}=\left\{\left(\begin{array}{cc}
\cos \theta & \sin \theta \\
-\sin \theta & \cos \theta
\end{array}\right): 0 \leq \theta<2 \pi\right\}=S O(2, \mathbb{R}), \\
& \mathcal{A}=\left\{\left(\begin{array}{cc}
r & 0 \\
0 & \frac{1}{r}
\end{array}\right): r>0\right\}, \\
& \mathcal{N}=\left\{\left(\begin{array}{cc}
1 & x \\
0 & 1
\end{array}\right): x \in \mathbb{R}\right\} .
\end{aligned}
$$

Theorem (Iwasawa Decomposition of $S p(2, \mathbb{R})=S L(2, \mathbb{R})$ ): We have the decomposition

$$
S p(2, \mathbb{R})=S L(2, \mathbb{R})=\mathcal{K} \mathcal{A N}
$$

i.e., every $S \in S p(2, \mathbb{R})=S L(2, \mathbb{R})$ has a unique representation $S=K A N$, where $K \in \mathcal{K}, A \in \mathcal{A}, N \in \mathcal{N}$.

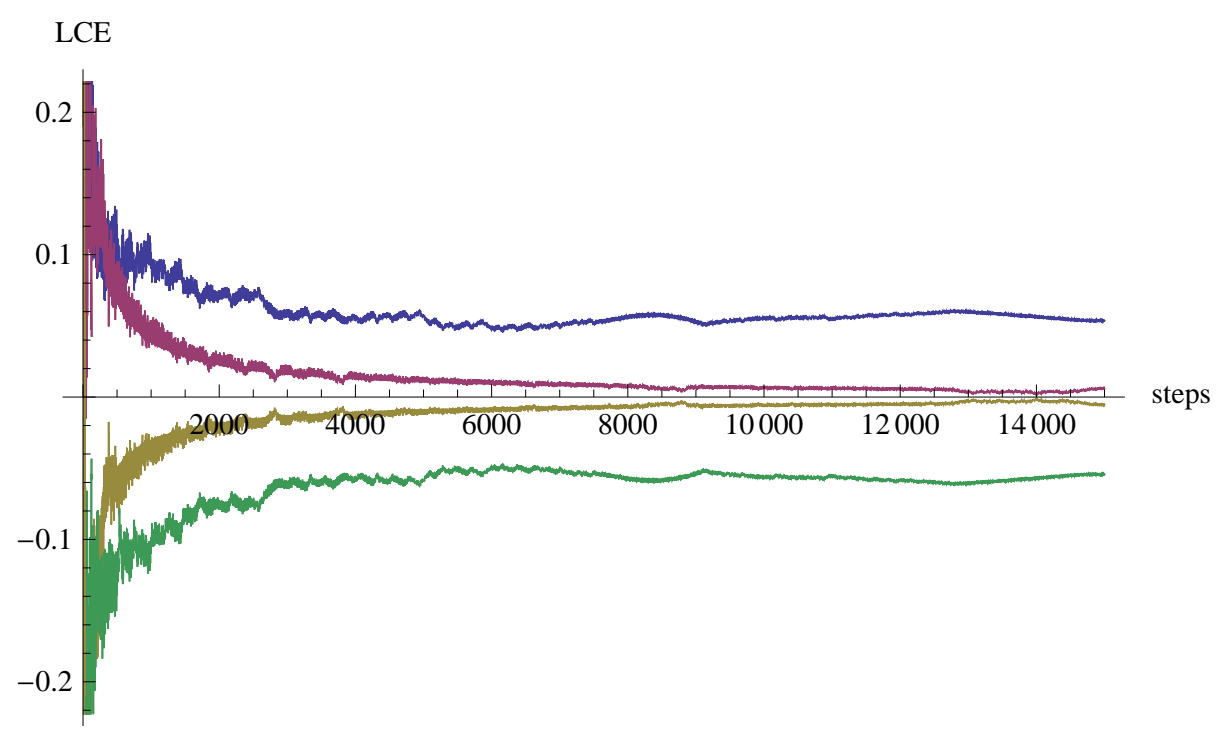

Fig. 8. 15000 steps of Lyapunov spectrum for the trajectory taken from region (I), we obtain $\lambda_{1} \approx 0.054, \lambda_{2} \approx 0, \lambda_{3} \approx 0 \lambda_{4} \approx-0.054$ 


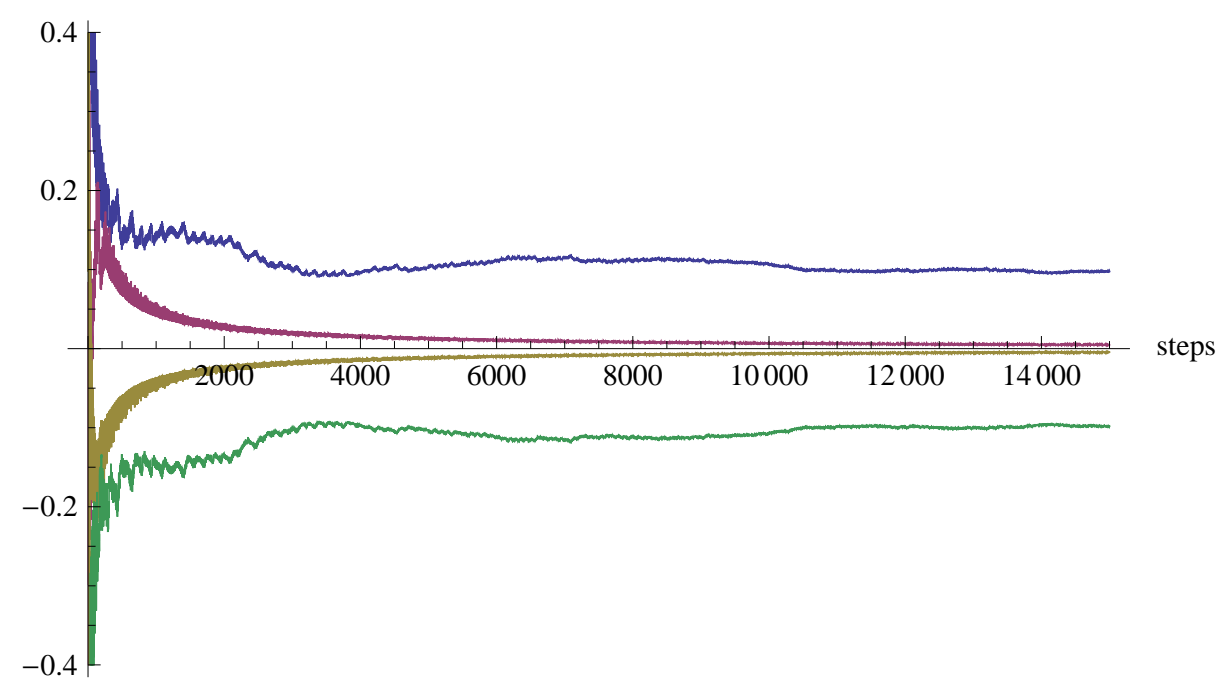

Fig. 9. 15000 steps of Lyapunov spectrum for the trajectory taken from region (II), we obtain $\lambda_{1} \approx 0.097, \lambda_{2} \approx 0, \lambda_{3} \approx 0 \lambda_{4} \approx-0.098$

Thus, in the two-dimensional case the Iwasawa decomposition is identical with the QR-decomposition, when $A$ and $N$ are subsumed to the upper triangular matrix $A N$.

Let us explicitly compute the symplectic local Lyapunov exponents in the most simple case of the harmonic oscillator with one degree of freedom. In this case, we have

$$
H(x, p)=\frac{p^{2}}{2}+\omega^{2} \frac{x^{2}}{2}
$$

and obtain

$$
Y(t):=\left(\begin{array}{cc}
\cos \omega t & \frac{1}{\omega} \sin \omega t \\
-\omega \sin \omega t & \cos \omega t
\end{array}\right)
$$

for the initial values $x(0)=1$ and $p(0)=0$.

For $\omega=2$, we compute for several $t$ the matrices $A N(t)$ of the Iwasawa decomposition and the matrices $\dot{A N}(t), U(t)$ as well as the symplectic local Lyapunov exponents (slLe) as follows:

\begin{tabular}{lll}
$t$ & $Y(t)$ & AN $(t)$ \\
\hline 1 & $\left(\begin{array}{cc}-0.416147 & -0.454649 \\
1.81859 & -0.416147\end{array}\right)$ & $\left(\begin{array}{cc}1.8656 & -0.304246 \\
0 & 0.53602\end{array}\right)$ \\
2001 & $\left(\begin{array}{cc}0.925273 & 0.18965 \\
-0.758602 & 0.925273\end{array}\right)$ & $\left(\begin{array}{cc}1.1965 & 0.930465 \\
0 & 0.835772\end{array}\right)$ \\
4001 & $\left(\begin{array}{cc}-0.934654 & 0.177779 \\
-0.711117 & -0.934654\end{array}\right)$ & $\left(\begin{array}{cc}1.17442 & -0.871222 \\
0 & 0.851483\end{array}\right)$ \\
6001 & $\left(\begin{array}{cc}0.439222 & -0.449189 \\
1.79676 & 0.439222\end{array}\right)$ & $\left(\begin{array}{cc}1.84966 & 0.319995 \\
0 & 0.540639\end{array}\right)$ \\
8001 & $\left(\begin{array}{cc}0.293436 & 0.477989 \\
-1.91196 & 0.293436\end{array}\right)$ & $\left(\begin{array}{cc}1.93434 & 6.51939 \\
0 & 0.516971\end{array}\right)$
\end{tabular}

$\left.\begin{array}{cc}t & \operatorname{Ain}(t) \\ 1 & \left(\begin{array}{cc}1.8656 & 0.304246 \\ 0 & 0.53602\end{array}\right) \\ 4001 & \left(\begin{array}{cc}1.1965 & -0.930465 \\ 0 & 0.835772\end{array}\right) \\ 6001 & \left(\begin{array}{cc}1.17442 & 0.871222 \\ 0 & 0.851483\end{array}\right) \\ 8001 & \left(\begin{array}{cc}1.84966 & -0.319995 \\ 0 & 0.540639\end{array}\right) \\ 0 & 0.516971\end{array}\right)$

$$
\begin{aligned}
& \mathrm{U}(\mathrm{t})=(\mathrm{AN}(\mathrm{t}))(\mathrm{AN}(\mathrm{t}))^{-1} \quad \text { slLe } \\
& \left(\begin{array}{cc}
-0.652329 & 2.70146
\end{array}\right) \quad-0.652329 \\
& 0.652329 \\
& \left(\begin{array}{cc}
-1.47089 & 3.93249 \\
0 & 1.47089
\end{array}\right) \quad \begin{array}{l}
-1.47089 \\
1.47089
\end{array} \\
& \left(\begin{array}{cc}
1.44566 & 3.95793 \\
0.0 & 1.44566
\end{array}\right. \\
& \begin{array}{llll}
0 . & -1.44566 & -1.44566
\end{array} \\
& \left(\begin{array}{cc}
0.692007 & 2.66167 \\
0.69
\end{array}\right) \quad 0.692007 \\
& \left.\begin{array}{lll}
0 . & -0.692007
\end{array}\right)-0.692007 \\
& \left(\begin{array}{cc}
-0.449827 & 84.0483 \\
0.0 & -0.449827
\end{array}\right. \\
& \text { o. } \quad 0.449827) \quad 0.449827
\end{aligned}
$$


Again we see that the symplectic local Lyapunov exponents occur in pairs, however, in the two-dimensional case this also holds for the identical QR local Lyapunov exponents.

\section{A. 2. A four-dimensional example}

In four dimensions, the Iwasawa decomposition of a symplectic matrix is not necessarily identical with the QRdecomposition of this matrix. In the main part of the article, using the Hénon-Heiles system as example we showed that this is the reason for the loss of the pairing property for QR local Lyapunov exponents, while the symplectic local Lyapunov exponents are always paired.

Here we consider a simpler non-chaotic example and show by explicit computations that the QR decomposition is in general not identical with the Iwasawa decomposition. For this purpose, consider the curve

$$
Y(t):=\left(\begin{array}{cccc}
\cosh (t) & \sinh (t) & a \sinh (t) & \sinh (t) \\
\sinh (t) & \cosh (t) & \sinh (t) & a \sinh (t) \\
0 & 0 & \cosh (t) & -\sinh (t) \\
0 & 0 & -\sinh (t) & \cosh (t)
\end{array}\right)
$$

in the symplectic group $S p(4, \mathbb{R})$. This curve satisfies $Y(0)=$ Id and depends on a parameter $a \in \mathbb{R}$. For $a:=0$, the QR-decomposition of each $Y(t), t \in \mathbb{R}$, is identical with the Iwasawa decomposition. However, for $a \neq 0$, the QRdecomposition and the Iwasawa decomposition of $Y(t)$ are different. For example, for $a:=1$ and $t:=1$

1. the QR-decomposition is

$$
\begin{gathered}
Y(t)= \\
=\left(\begin{array}{cccc}
0.79555 & -0.60589 & 0 & 0 \\
0.60589 & 0.79555 & 0 & 0 \\
0 & 0 & 0.79555 & 0.60589 \\
0 & 0 & -0.60589 & 0.79555
\end{array}\right) \\
\left(\begin{array}{cccc}
1.93964 & 1.86986 & 1.64697 & 1.64697 \\
0 & 0.51556 & 0.22289 & 0.22289 \\
0 & 0 & 1.93964 & -1.86986 \\
0 & 0 & 0 & 0.51556
\end{array}\right),
\end{gathered}
$$

and $Q$ does not preserve the standard symplectic structure $J$,

2. the Iwasawa decomposition $Y=K \cdot A N$ is

$$
\begin{gathered}
Y(t)= \\
=\left(\begin{array}{cccc}
0.79555 & -0.60589 & 0 & 0 \\
0.60589 & 0.79555 & 0 & 0 \\
0 & 0 & 0.79555 & -0.60589 \\
0 & 0 & 0.60589 & 0.79555
\end{array}\right) \\
\left(\begin{array}{cccc}
1.93964 & 1.86986 & 1.64697 & 1.64697 \\
0 & 0.51556 & 0.22289 & 0.22289 \\
0 & 0 & 0.51556 & 0 \\
0 & 0 & -1.86986 & 1.93964
\end{array}\right),
\end{gathered}
$$

and $K$ preserves the standard symplectic structure $J$.
Note that in this simple example the QR and symplectic local Lyapunov exponents are identical. In fact, $Y(t)$ solves $\dot{Y}=A Y$ for the constant matrix

$$
A:=\left(\begin{array}{cccc}
0 & 1 & a & 1 \\
1 & 0 & 1 & a \\
0 & 0 & 0 & -1 \\
0 & 0 & -1 & 0
\end{array}\right)=J\left(\begin{array}{cccc}
0 & 0 & 0 & 1 \\
0 & 0 & 1 & 0 \\
0 & 1 & a & 1 \\
1 & 0 & 1 & a
\end{array}\right) .
$$

Particularly, this decoupled ODE is the linearization of the system to the Hamiltonian $H\left(x_{1}, x_{2}, x_{3}, x_{4}\right):=x_{1} x_{4}+$ $x_{2} x_{3}+\frac{a}{2}\left(x_{3}^{2}+x_{4}^{2}\right)+x_{3} x_{4}$. As the Hamiltonian is quadratic, the Hamiltonian system is linear, and there is not only no chaos, but as the equations are decoupled, the system is even integrable. Thus, it is no wonder that $\mathrm{QR}$ and symplectic local Lyapunov exponents coincide in this case.

However, Figures 3 and 4 in the main part of this article show that the QR local Lyapunov exponents can be different from the symplectic local Lyapunov exponents.

\section{References}

[1] V.I. Arnold, Mathematical Methods of Classical Mechanics, 2nd Ed., Springer, New York 1980.

[2] G. Benettin, L. Galgani, A. Giorgilli, J.-M. Strelcyn, Lyapunov characteristic exponents for smooth dynamical systems and for Hamiltonian systems; a method for computing all of them, Part 1: Theory, Meccanica 15(1), 9-20 (1980).

[3] L. Barreira, Ya. Pesin, Lectures on Lyapunov exponents and smooth ergodic theory, in: Proceedings of symposia in pure mathematics, AMS, Providence, RI, 3-90 (2001).

[4] M. Benzi, N. Razouk, On the Iwasawa decomposition of a symplectic matrix, Applied Mathematics Letters 20, 260265 (2007).

[5] L.A. Bunimovich, Mushrooms and other billiards with divided phase space, Chaos 11(4), 802-808 (2001).

[6] Farouk Cherif, Theoretical Computation of Lyapunov Exponents for Almost Periodic Hamiltonian Systems, IAENG International Journal of Applied Mathematics 41:1, IJAM_ 41_1_02 (2011).

[7] J.P. Eckmann, D. Ruelle, Ergodic theory of chaos and strange attractors, Reviews of Modern Physics 57, 617-656 (1985).

[8] F. Ginelli, P. Poggi, A. Turchi, H. Chaté, R. Livi, A. Politi, Characterizing dynamics with covariant Lyapunov vectors, Phys. Rev. Lett. 99, 130601 (2007).

[9] Wm.G. Hoover, C.G. Hoover, Instantaneous Pairing of Lyapunov Exponents in Chaotic Hamiltonian Dynamics and the 2017 Ian Snook Prizes, CMST 23(1), 73-79 (2017).

[10] J.E. Marsden, T.S. Ratiu, Introduction to mechanics and symmetry Springer, 1994.

[11] H.A. Posch, Symmetry Properties of Orthogonal and Covariant Lyapunov Vectors and Their Exponents, Journal of Physics A 46, 254006 (2013).

[12] P. Sawyer, Computing the Iwasawa decomposition of the classical Lie groups of noncompact type using the QR decomposition, Linear Algebra and its Applications 493, 573-579 (2016).

[13] I.I. Shevchenko, A.V. Mel'nikov, Lyapunov Exponents in the Hénon-Heiles Problem, JETP Letters 77(12), 642-646 (2003).

[14] W. Li, S. Shi, Non-integrability of Hénon-Heiles system, Celest. Mech. Dyn. Astr. 109, 1-12 (2011). 
[15] L. Zachilas, A review study of the 3-particle Toda lattice and higher-order truncations: The odd-order cases (part I), International Journal of Bifurcation and Chaos 20(10), 3007-3064 (2010).
[16] L. Zachilas, A review study of the 3-particle Toda lattice and higher-order truncations: The even-order cases (part II), International Journal of Bifurcation and Chaos 20(11), 3391-3441 (2010).
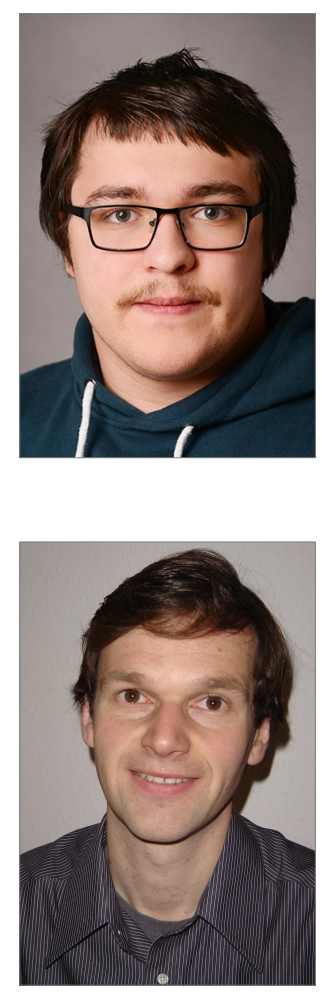

Timo Gerald Hofmann is a 17-year-old student at Leipzig Ostwald Grammar School in class eleven. He takes A-level advanced courses in Mathematics, Physics and Chemistry. Besides his schoolwork he has attended lectures in Mathematics (Analysis I \& II, Differential Equations, Nonlinear Programming, Numerical Mathematics) at Leipzig University of Applied Sciences (HTWK) since 2014. He has taken part in various Mathematics contests since class 6 and won several first prizes at Mathematical Olympiads in Saxony, and a first and two third prizes at the German National Mathematics Competition. In 2016 and 2017 he took part in the International Olympiad of Metropolises in Moscow, and won two third prizes with his team. Furthermore, he has taken part in Physics contests, and won first and second prizes at the German National Physics Competition in 2016 and 2017, and qualified for the German contest for the International Physics Olympiad in 2017 and 2018. Moreover, he is interested in chess and won the second place with his team at the German Youth Team Chess Championships in 2016.

Jochen Merker has been professor of "Analysis and Optimization" at the HTWK Leipzig since 2015. He received his PhD in Mathematics from the University of Hamburg in 2005. Afterwards he worked as a Postdoc in "Applied Analysis" at the University of Rostock, received his Habilitation in 2012 and became a professor at the Applied University of Stralsund in 2013, before he moved to Leipzig. His research focuses on doubly nonlinear evolution equations, degenerate and singular parabolic PDEs and Hamiltonian systems on manifolds. 